ANNALES

POLONICI MATHEMATICI

LXXVII.3 (2001)

\title{
Cross theorem
}

\section{by Marek Jarnicki (Kraków) and Peter Pflug (Oldenburg)}

Abstract. Let $D, G \subset \mathbb{C}$ be domains, let $A \subset D, B \subset G$ be locally regular sets, and let $X:=(D \times B) \cup(A \times G)$. Assume that $A$ is a Borel set. Let $M$ be a proper analytic subset of an open neighborhood of $X$. Then there exists a pure 1-dimensional analytic subset $\widehat{M}$ of the envelope of holomorphy $\widehat{X}$ of $X$ such that any function separately holomorphic on $X \backslash M$ extends to a holomorphic function on $\widehat{X} \backslash \widehat{M}$. The result generalizes special cases which were studied in [Ökt 1998], [Ökt 1999], and [Sic 2000].

1. Introduction. Main result. For domains $D \subset \mathbb{C}^{n}, G \subset \mathbb{C}^{m}$ and non-pluripolar subsets $A \subset D, B \subset G$, define the cross

$$
X=\mathbb{X}(D, A ; G, B):=(D \times B) \cup(A \times G)
$$

(notice that $X$ is connected). Let $U \subset D \times G$ be an open connected neighborhood of $X$ and let $M$ be an analytic subset of $U, M \neq U$. Put

$$
\begin{aligned}
& M_{z}:=\{w \in G:(z, w) \in M\}, \quad z \in D, \\
& M^{w}:=\{z \in D:(z, w) \in M\}, \quad w \in G .
\end{aligned}
$$

We say that a function $f: X \backslash M \rightarrow \mathbb{C}$ is separately holomorphic on $X \backslash M$ $\left(f \in \mathcal{O}_{\mathrm{s}}(X \backslash M)\right)$ if

$$
\forall_{z \in A, M_{z} \neq G}: f(z, \cdot) \in \mathcal{O}\left(G \backslash M_{z}\right), \quad \forall_{w \in B, M^{w} \neq D}: f(\cdot, w) \in \mathcal{O}\left(D \backslash M^{w}\right) .
$$

For an open set $\Omega \subset \mathbb{C}^{n}$ and $A \subset \Omega$ put

$$
h_{A, \Omega}:=\sup \{u: u \in \mathcal{P} \mathcal{S H}(\Omega), u \leq 1 \text { on } \Omega, u \leq 0 \text { on } A\},
$$

where $\mathcal{P} \mathcal{S H}(\Omega)$ denotes the set of all functions plurisubharmonic on $\Omega$.

2000 Mathematics Subject Classification: 32D15, 32D10.

Key words and phrases: separately holomorphic, locally regular, locally pluriregular, pluriregular, cross.

The paper, inspired by Siciak's lectures on [Sic 2000], was started during the stay of the second author at the Jagiellonian University in Kraków (October 2000) and it was finished during the stay of the first author at the Carl von Ossietzky University in Oldenburg (November 2000). 
Define

$$
\omega_{A, \Omega}:=\lim _{k \rightarrow \infty} h_{A \cap \Omega_{k}, \Omega_{k}}^{*},
$$

where $\left(\Omega_{k}\right)_{k=1}^{\infty}$ is a sequence of relatively compact open sets $\Omega_{k} \subset \Omega_{k+1} \subset \subset$ $\Omega$ with $\bigcup_{k=1}^{\infty} \Omega_{k}=\Omega\left(h^{*}\right.$ denotes the upper semicontinuous regularization of $h)$. Note that the definition is independent of the approximation sequence $\left(\Omega_{k}\right)_{k=1}^{\infty}$.

For a cross $(*)$ put

$$
\widehat{X}:=\left\{(z, w) \in D \times G: \omega_{A, D}(z)+\omega_{B, G}(w)<1\right\} .
$$

We say that a subset $A \subset \mathbb{C}^{n}$ is locally pluriregular if $h_{A \cap \Omega, \Omega}^{*}(a)=0$ for any $a \in A$ and for any open neighborhood $\Omega$ of $a$ (in particular, $A \cap \Omega$ is non-pluripolar). As always, if $n=1$, then we say that $A$ is locally "regular" instead of "pluriregular".

The main result of the paper is the following

Theorem 1. Let $D, G \subset \mathbb{C}$ be domains, let $A \subset D, B \subset G$ be locally regular sets, and let $X:=(D \times B) \cup(A \times G)$. Assume that $A$ is a Borel set. Let $M$ be a proper analytic subset of an open connected neighborhood $U$ of $X$. Then there exists a pure 1-dimensional analytic subset $\widehat{M}$ of $\widehat{X}$ $(\widehat{X}$ is given by $(* *))$ such that for any $f \in \mathcal{O}_{\mathrm{s}}(X \backslash M)$ there exists exactly one $\widehat{f} \in \mathcal{O}(\widehat{X} \backslash \widehat{M})$ with $\widehat{f}=f$ on $X \backslash(M \cup \widehat{M})$.

Moreover, if $U=\widehat{X}$ and $M$ is pure 1-dimensional, then the above condition is satisfied by $\widehat{M}:=M$.

REmark. Consider the following general problem. Let $D_{j} \subset \mathbb{C}^{n_{j}}$ be a domain of holomorphy and let $A_{j} \subset D_{j}$ be a locally pluriregular Borel set, $j=1, \ldots, N$. Define the generalized cross

$X:=\left(A_{1} \times \ldots \times A_{N-1} \times D_{N}\right) \cup \ldots \cup\left(D_{1} \times A_{2} \times \ldots \times A_{N}\right) \subset \mathbb{C}^{n_{1}} \times \ldots \times \mathbb{C}^{n_{N}}$.

Let $U \subset D_{1} \times \ldots \times D_{N}$ be a connected neighborhood of $X$ and let $M \subset U$ be a proper analytic set. A function $f: X \backslash M \rightarrow \mathbb{C}$ is said to be separately holomorphic $\left(f \in \mathcal{O}_{\mathrm{S}}(X \backslash M)\right)$ if for any $\left(a_{1}, \ldots, a_{N}\right) \in A_{1} \times \ldots \times A_{N}$ and $k \in\{1, \ldots, N\}$ the function $f\left(a_{1}, \ldots, a_{k-1}, \cdot, a_{k+1}, \ldots, a_{N}\right)$ is holomorphic in the domain $\left\{z_{k} \in D_{k}:\left(a_{1}, \ldots, a_{k-1}, z_{k}, a_{k+1}, \ldots, a_{N}\right) \notin M\right\}$. Define $\widehat{X}:=\left\{\left(z_{1}, \ldots, z_{N}\right) \in D_{1} \times \ldots \times D_{N}: \omega_{A_{1}, D_{1}}\left(z_{1}\right)+\ldots+\omega_{A_{N}, D_{N}}\left(z_{N}\right)<1\right\}$.

Conjecture $\left({ }^{1}\right)$. There exists a pure 1-codimensional analytic subset $\widehat{M} \subset \widehat{X}$ such that for any $f \in \mathcal{O}_{\mathrm{s}}(X \backslash M)$ there exists an $\widehat{f} \in \mathcal{O}(\widehat{X} \backslash \widehat{M})$ with $\widehat{f}=f$ on $X \backslash(M \cup \widehat{M})$. Moreover, $\widehat{M}=M$ if $U=\widehat{X}$ and $M$ is pure 1-codimensional. Compare also [Ökt 1999] (for $N=2$ and $U=\widehat{X}$ ).

$\left({ }^{1}\right)$ Added in proof: Cf. M. Jarnicki, P. Pflug, An extension theorem for separately holomorphic functions with singularities, IMUJ Preprint 2001/27 (2001). 
Theorem 1 solves the case $N=2, n_{1}=n_{2}=1$.

J. Siciak [Sic 2000] solved the following case: $n_{1}=\ldots=n_{N}=1, D_{1}=$ $\ldots=D_{N}=\mathbb{C}, M=P^{-1}(0)$, where $P$ is a non-zero polynomial of $N$ complex variables; the special subcase $N=2, P(z, w):=z-w$ had been previously studied in [Ökt 1998].

The case studied in [Sic 2000] is the only known case with $n_{1}+\ldots+n_{N}$ $>2$. In the general case, the answer is not known even if $U=\widehat{X}$ and $M$ is pure 1-codimensional.

2. Auxiliary results. The following lemma gathers a few standard results which will be used in what follows.

LEMmA 2 (cf. [Kli 1991], [Jar-Pfl 2000], §3.5). (a) Let $\Omega \subset \mathbb{C}^{n}$ be a bounded open set and let $A \subset \Omega$. Then:

- If $P \subset \mathbb{C}^{n}$ is pluripolar, then $h_{A \backslash P, \Omega}^{*}=h_{A, \Omega}^{*}$.

- $h_{A_{k} \cap \Omega_{k}, \Omega_{k}} \searrow h_{A, \Omega}^{*}$ (pointwise on $\Omega$ ) for any sequence of open sets $\Omega_{k} \nearrow \Omega$ and any sequence $A_{k} \nearrow A$.

- $\omega_{A, \Omega}=h_{A, \Omega}^{*}$.

- The following two conditions are equivalent:

(i) for any connected component $S$ of $\Omega$ the set $A \cap S$ is non-pluripolar;

(ii) $h_{A, \Omega}^{*}(z)<1$ for any $z \in \Omega$.

- If $A$ is non-pluripolar, $0<\alpha<1$, and $\Omega_{\alpha}:=\left\{z \in \Omega: h_{A, \Omega}^{*}(z)<\alpha\right\}$, then for any connected component $S$ of $\Omega_{\alpha}$ the set $A \cap S$ is non-pluripolar (in particular, $A \cap S \neq \emptyset$ ).

(b) Let $\Omega \subset \mathbb{C}^{n}$ be an open set and let $A \subset \Omega$. Then:

- $\omega_{A, \Omega} \in \mathcal{P S H}(\Omega)$.

- If $A$ is locally pluriregular, then $\omega_{A, \Omega}(a)=0$ for any $a \in A$.

- If $P \subset \mathbb{C}^{n}$ is pluripolar, then $\omega_{A \backslash P, \Omega}=\omega_{A, \Omega}$.

- If $A$ is locally pluriregular and $P \subset \mathbb{C}^{n}$ is pluripolar, then $A \backslash P$ is locally pluriregular.

(c) Let $X=\mathbb{X}(D, A ; G, B)$ be a cross as in $(*)$. Then:

- If $A$ and $B$ are locally pluriregular, then $X \subset \widehat{X}$.

- If $D$ and $G$ are domains of holomorphy, then $\widehat{X}$ is a region of holomorphy.

Lemma 3. Let $X=\mathbb{X}(D, A ; G, B)$ be a cross as in $(*)$. If $A$ and $B$ are locally pluriregular, then $\widehat{X}$ is a domain.

Proof. It suffices to show that for any approximation sequences $D_{k} \nearrow D$, $G_{k} \nearrow G$ of relatively compact subdomains with $A \cap D_{k} \neq \emptyset, B \cap G_{k} \neq \emptyset$, 
$k \in \mathbb{N}$, the sets

$$
\widehat{X}_{k}:=\left\{(z, w) \in D_{k} \times G_{k}: h_{A \cap D_{k}, D_{k}}^{*}(z)+h_{B \cap G_{k}, G_{k}}^{*}(w)<1\right\}, \quad k \in \mathbb{N},
$$

are connected. Thus, we may assume that $D$ and $G$ are bounded. Since the cross $X$ is connected and contained in $\widehat{X}$, we only need to prove that for any $\left(z_{0}, w_{0}\right) \in \widehat{X}$, each connected component of the fiber

$$
\widehat{X}^{w_{0}}:=\left\{z \in D:\left(z, w_{0}\right) \in \widehat{X}\right\}=\left\{z \in D: h_{A, D}^{*}(z)<1-h_{B, G}^{*}\left(w_{0}\right)\right\}
$$

intersects $A$. If $h_{B, G}^{*}\left(w_{0}\right)=0$, then $\widehat{X}^{w_{0}}=D$. If $h_{B, G}^{*}\left(w_{0}\right)>0$, then we apply Lemma $2(\mathrm{a})$.

Theorem 4 (Classical cross theorem, cf. [Ngu-Zer 1991]). Let $X=$ $\mathbb{X}(D, A ; G, B)$ be as in $(*)$. Assume that:

- $D, G$ are domains of holomorphy,

- A, $B$ are locally pluriregular,

- A is a Borel set.

Then for any $f \in \mathcal{O}_{\mathrm{s}}(X)$ there exists exactly one $\widehat{f} \in \mathcal{O}(\widehat{X})$ with $\widehat{f}=f$ on $X$.

TheOREM 5 (Dloussky-Grauert-Remmert theorem, cf. [Jar-Pfl 2000], $\S 3.4)$. Let $\Omega \subset \mathbb{C}^{n}$ be a domain and let $M$ be an analytic subset of $\Omega$. Let $\widehat{\Omega}$ be the envelope of holomorphy of $\Omega$ (univalent or not). Then there exists a pure 1-codimensional analytic subset $\widehat{M} \subset \widehat{\Omega}$ uch that for any $g \in \mathcal{O}(\Omega \backslash M)$ there exists $\widehat{g} \in \mathcal{O}(\widehat{\Omega} \backslash \widehat{M})$ with $\widehat{g}=g$ on $\Omega \backslash(M \cup \widehat{M})$.

If , moreover, $M=\Omega \cap \widetilde{M}$, where $\widetilde{M}$ is a pure 1-codimensional analytic subset of $\widehat{\Omega}$, then the above condition is satisfied by $\widehat{M}:=\widetilde{M}$.

Lemma 6. Let $D, G \subset \mathbb{C}$ be domains, let $A \subset D, B \subset G$ be locally regular sets, and let $X:=\mathbb{X}(D, A ; G, B)$. Let $M$ be a proper analytic subset of an open connected neighborhood $U$ of $X$. Assume that $A^{\prime} \subset A, B^{\prime} \subset B$ are such that:

- $A \backslash A^{\prime}$ and $B \backslash B^{\prime}$ are polar (in particular, $A^{\prime}, B^{\prime}$ are also locally regular $)$,

- $M_{z} \neq G$ for any $z \in A^{\prime}$ and $M^{w} \neq D$ for any $w \in B^{\prime}$.

(a) If $f \in \mathcal{O}_{\mathrm{s}}(X \backslash M)$ and $f=0$ on $\left(A^{\prime} \times B^{\prime}\right) \backslash M$, then $f=0$ on $X \backslash M$.

(b) If $g \in \mathcal{O}(U \backslash M)$ and $g=0$ on $\left(A^{\prime} \times B^{\prime}\right) \backslash M$, then $g=0$ on $U \backslash M$.

Proof. (a) Take a point $\left(a_{0}, b_{0}\right) \in X \backslash M$. We may assume that $a_{0} \in A$. Since $A \backslash A^{\prime}$ is polar, there exists a sequence $\left(a_{k}\right)_{k=1}^{\infty} \subset A^{\prime}$ such that $a_{k} \rightarrow a_{0}$. The set $Q:=\bigcup_{k=0}^{\infty} M_{a_{k}}$ is at most countable. Consequently, the set $B^{\prime \prime}:=$ $B^{\prime} \backslash Q$ is non-polar. We have $f\left(a_{k}, w\right)=0$ for all $w \in B^{\prime \prime}, k=1,2, \ldots$ Hence $f\left(a_{0}, w\right)=0$ for any $w \in B^{\prime \prime}$. Finally, $f\left(a_{0}, w\right)=0$ on $G \backslash M_{a_{0}} \ni b_{0}$. 
(b) Take an $a_{0} \in A^{\prime}$. Since $M_{a_{0}} \neq G$, there exists a $b_{0} \in B^{\prime} \backslash M_{a_{0}}$. Let $P=\Delta_{a_{0}}(r) \times \Delta_{b_{0}}(r) \subset U \backslash M\left(\Delta_{z_{0}}(r)\right.$ denotes the disc with center $z_{0}$ and radius $r$ ). Then $g(\cdot, w)=0$ on $A^{\prime} \cap \Delta_{a_{0}}(r)$ for any $w \in B^{\prime} \cap \Delta_{b_{0}}(r)$. The set $A^{\prime} \cap \Delta_{a_{0}}(r)$ is non-polar. Hence $g(\cdot, w)=0$ on $\Delta_{a_{0}}(r)$ for any $w \in B^{\prime} \cap \Delta_{b_{0}}(r)$. By the same argument for the second variable we get $g=0$ on $P$ and, consequently, on $U$.

\section{Proof of the main theorem}

STEP 1. Fix sequences $D_{k} \nearrow D, G_{k} \nearrow G$ of relatively compact subdomains with $D_{k} \subset \subset D_{k+1}, A \cap D_{k} \neq \emptyset, G_{k} \subset \subset G_{k+1}, B \cap G_{k} \neq \emptyset, k \in \mathbb{N}$.

For any $a \in A$ such that $M_{a} \neq G$ we perform the following construction:

Fix a $k \in \mathbb{N}, k \geq 2$. Let $M_{a} \cap G_{k}=\left\{b_{1}, \ldots, b_{N}\right\}$. Fix domains $G^{\prime}=G_{a, k}^{\prime}$, $G^{\prime \prime}=G_{a, k}^{\prime \prime}$ such that $G_{k-1} \subset \subset G^{\prime \prime} \subset \subset G^{\prime} \subset \subset G_{k}$ and $b_{1}, \ldots, b_{N} \in G^{\prime \prime}$. Take positive numbers $\delta, \varepsilon, \eta>\varepsilon$ such that

- $\Delta_{a}(\delta) \subset \subset D$,

- $\Delta_{b_{j}}(\eta) \subset \subset G^{\prime \prime}, j=1, \ldots, N$,

- $\bar{\Delta}_{b_{i}}(\eta) \cap \bar{\Delta}_{b_{j}}(\eta)=\emptyset, i, j=1, \ldots, N, i \neq j$,

- $M \cap\left(\Delta_{a}(\delta) \times G^{\prime}\right) \subset \bigcup_{j=1}^{N} \Delta_{a}(\delta) \times \Delta_{b_{j}}(\varepsilon)$,

- $B \cap V^{\prime \prime} \neq \emptyset$, where $V^{\prime \prime}:=G^{\prime \prime} \backslash \bigcup_{j=1}^{N} \bar{\Delta}_{b_{j}}(\eta)$.

Define $V^{\prime}:=G^{\prime} \backslash \bigcup_{j=1}^{N} \bar{\Delta}_{b_{j}}(\varepsilon)$. Note that $V^{\prime \prime} \subset \subset V^{\prime}$. Consider the cross $Y=Y_{a, k}:=\mathbb{X}\left(\Delta_{a}(\delta), A \cap \Delta_{a}(\delta) ; V^{\prime}, B \cap V^{\prime}\right)$.

Fix an $f \in \mathcal{O}_{\mathrm{s}}(X \backslash M)$. Then $f \in \mathcal{O}_{\mathrm{s}}(Y)$. By Theorem 4, the function $f$ extends holomorphically to $\widehat{Y} \supset\{a\} \times V^{\prime}$. Consequently, there exists $0<\widehat{\delta}<\delta$ such that $f$ is holomorphic in $\Delta_{a}(\widehat{\delta}) \times V^{\prime \prime}$.

SteP 2. Suppose that for some $j \in\{1, \ldots, N\}$ we have

$$
M \cap\left(\Delta_{a}(\delta) \times \Delta_{b_{j}}(\varepsilon)\right) \subset\left\{\left(z, \varphi_{j}(z)\right): z \in \Delta_{a}(\delta)\right\},
$$

where $\varphi_{j}: \Delta_{a}(\delta) \rightarrow \Delta_{b_{j}}(\varepsilon)$ is holomorphic.

We will prove that for sufficiently small $\delta^{\prime}>0$ the function $f$ extends holomorphically to $\left(\Delta_{a}\left(\delta^{\prime}\right) \times \Delta_{b_{j}}(\eta)\right) \backslash\left\{\left(z, \varphi_{j}(z)\right): z \in \Delta_{a}\left(\delta^{\prime}\right)\right\}$.

Indeed, by Step 1, there exists $\eta^{\prime}>\eta$ such that the function $f$ extends holomorphically to $\Delta_{a}(\widehat{\delta}) \times\left(\Delta_{b_{j}}\left(\eta^{\prime}\right) \backslash \bar{\Delta}_{b_{j}}(\eta)\right)$. Using the biholomorphism

$$
\Delta_{a}(\delta) \times \mathbb{C} \ni(z, w) \mapsto\left(z, w-\varphi_{j}(z)\right) \in \Delta_{a}(\delta) \times \mathbb{C},
$$

we reduce the problem to the case where $\varphi_{j} \equiv 0$. Thus we have the following problem:

Let $\Delta(r):=\Delta_{0}(r)$. Given a function $f$ holomorphic on $\Delta_{a}(\varrho) \times P$, where $P:=\Delta(R) \backslash \bar{\Delta}(r)$, such that $f(z, \cdot) \in \mathcal{O}(\Delta(R) \backslash\{0\})$ for any $z \in A \cap \Delta_{a}(\varrho)$, we prove that $f$ extends holomorphically to $\Delta_{a}(\varrho) \times(\Delta(R) \backslash\{0\})$. 
Indeed, consider the cross

$$
Y:=\mathbb{X}\left(\Delta_{a}(\varrho), A \cap \Delta_{a}(\varrho) ; \Delta(R) \backslash\{0\}, P\right) .
$$

By Theorem 4 , the function $f$ extends to $\widehat{Y}$. It remains to observe that $\widehat{Y}=\Delta_{a}(\varrho) \times(\Delta(R) \backslash\{0\})$ (because $\left.h_{P, \Delta(R) \backslash\{0\}}^{*} \equiv 0\right)$.

In particular, if

$$
M \cap\left(\Delta_{a}(\delta) \times \Delta_{b_{j}}(\varepsilon)\right)=\left\{\left(z, \varphi_{j}(z)\right): z \in \Delta_{a}(\delta)\right\},
$$

where $\varphi_{j}: \Delta_{a}(\delta) \rightarrow \Delta_{b_{j}}(\varepsilon)$ is holomorphic, for all $j=1, \ldots, N$, then there exists $\delta^{\prime}>0$ such that $f$ extends holomorphically to $\left(\Delta_{a}\left(\delta^{\prime}\right) \times G^{\prime \prime}\right) \backslash M \supset$ $\left(\Delta_{a}\left(\delta^{\prime}\right) \times G_{k-1}\right) \backslash M$.

SteP 3. Suppose that for some $j \in\{1, \ldots, N\}$ we have

$$
M \cap\left(\Delta_{a}(\delta) \times \Delta_{b_{j}}(\varepsilon)\right)=\left\{\left(a, b_{j}\right)\right\} .
$$

By Step $2\left(\right.$ with $\varphi_{j} \equiv b_{j}$ ) the function $f$ extends holomorphically to $\Delta_{a}\left(\delta^{\prime}\right) \times$ $\left(\Delta_{b_{j}}(\eta) \backslash\left\{b_{j}\right\}\right)$ for some small $\delta^{\prime}>0$. On the other hand, we know that $f$ is separately holomorphic on $Z:=\left(\Delta_{a}(\delta) \backslash\{a\}\right) \times \Delta_{b_{j}}(\varepsilon)$. Consequently, $f$ is holomorphic on $Z$. Hence $f$ is holomorphic on $\left(\Delta_{a}\left(\delta^{\prime}\right) \times \Delta_{b_{j}}(\varepsilon)\right) \backslash\left\{\left(a, b_{j}\right)\right\}$. Thus $\left(a, b_{j}\right)$ is a removable singularity of $f$.

In virtue of the above remark, we may assume that $M$ is pure 1-dimensional.

STEP 4. Let $A^{\prime}$ denote the set of all $a \in A$ such that for each $k \geq 2$ either there exists $\delta>0$ such that $M \cap\left(\Delta_{a}(\delta) \times G_{k}\right)=\emptyset$ or the construction from Step 1 may be performed in such a way that for each $j \in\{1, \ldots, N\}$,

$$
M \cap\left(\Delta_{a}(\delta) \times \Delta_{b_{j}}(\varepsilon)\right)=\left\{\left(z, \varphi_{j}(z)\right): z \in \Delta_{a}(\delta)\right\}
$$

where $\varphi_{j}: \Delta_{a}(\delta) \rightarrow \Delta_{b_{j}}(\varepsilon)$ is holomorphic (cf. Step 2). Then $A \backslash A^{\prime}$ is at most countable. Indeed, write

$$
M=\bigcup_{j=1}^{\infty}\left\{(z, w) \in P_{j}: g_{j}(z, w)=0\right\},
$$

where $P_{j} \subset \subset U$ is a polydisc and $g_{j}$ is a defining function for $M \cap P_{j}$ (cf. [Chi 1989], § 2.9). Put $S_{j}:=\left\{(z, w) \in P_{j}: g_{j}(z, w)=\frac{\partial g_{j}}{\partial w}(z, w)=0\right\}$. Observe that if $\left(z_{0}, w_{0}\right) \in\left(M \cap P_{j}\right) \backslash S_{j}$, then there exists a small polydisc $Q=Q^{\prime} \times Q^{\prime \prime} \subset \subset P_{j}$ with center at $\left(z_{0}, w_{0}\right)$ such that $M \cap Q$ is the graph of a holomorphic function $\varphi: Q^{\prime} \rightarrow Q^{\prime \prime}$.

The projection $\operatorname{pr}_{z}\left(S_{j}\right)$ is at most countable. Indeed, we only need to prove that $\operatorname{pr}_{z}\left(S_{j}^{\prime}\right)$ is at most countable, where $S_{j}^{\prime}$ is the union of all 1dimensional irreducible components of $S_{j}$. Let $S$ be such an irreducible component. We will show that $S$ projects onto one point. Take $\left(z_{1}, w_{1}\right),\left(z_{2}, w_{2}\right)$ $\in S$. We want to show that $z_{1}=z_{2}$. It suffices to consider the case where $\left(z_{1}, w_{1}\right),\left(z_{2}, w_{2}\right)$ are regular points of $S$. Let $\psi=\left(\psi_{1}, \psi_{2}\right):[0,1] \rightarrow \operatorname{Reg}(S)$ 
be a $\mathcal{C}^{1}$-curve with $\psi(0)=\left(z_{1}, w_{1}\right), \psi(1)=\left(z_{2}, w_{2}\right)$. Note that $\frac{\partial g_{j}}{\partial z}(z, w) \neq 0$ for $(z, w) \in \operatorname{Reg}(S)$ (because $g_{j}$ is a defining function). We have

$$
0=\frac{\partial\left(g_{j} \circ \psi\right)}{\partial t}(t)=\frac{\partial g_{j}}{\partial z}(\psi(t)) \psi_{1}^{\prime}(t), \quad t \in[0,1] .
$$

Thus $\psi_{1}^{\prime} \equiv 0$. In particular, $z_{1}=z_{2}$.

Consequently, $A \backslash A^{\prime} \subset \bigcup_{j=1}^{\infty} \operatorname{pr}_{z}\left(S_{j}\right)$ is at most countable.

STEP 5. Let $B^{\prime}$ be constructed analogously to $A^{\prime}$ with respect to the second variable. Put $X^{\prime}:=\mathbb{X}\left(D, A^{\prime} ; G, B^{\prime}\right)$.

By Step 2 (and Lemma 6), for any $k \in \mathbb{N}$ and any $\xi=(a, b) \in\left(A^{\prime} \cap D_{k}\right)$ $\times\left(B^{\prime} \cap G_{k}\right)$ there exists $\varrho=\varrho_{\xi, k}>0$ such that for each $f \in \mathcal{O}_{\mathrm{s}}(X \backslash M)$ there exists $\widetilde{f}=\widetilde{f}_{\xi, k} \in \mathcal{O}\left(\Omega_{\xi, k} \backslash M\right)$ with $\widetilde{f}=f$ on $X \cap \Omega_{\xi, k} \backslash M$, where

$$
\begin{aligned}
\Omega_{\xi, k} & :=\mathbb{X}\left(D_{k}, \Delta_{a}(\varrho) ; G_{k}, \Delta_{b}(\varrho)\right) \\
& =\left(\Delta_{a}(\varrho) \times G_{k}\right) \cup\left(D_{k} \times \Delta_{b}(\varrho)\right) \subset U \cap\left(D_{k} \times G_{k}\right) .
\end{aligned}
$$

We may always assume that $\varrho_{\xi, k+1} \leq \varrho_{\xi, k}$. By Lemma $6, \widetilde{f}_{\xi, k+1}=\widetilde{f}_{\xi, k}$ on $\Omega_{\xi, k+1} \cap \Omega_{\xi, k} \backslash M$. Define

$$
\Omega:=\bigcup_{k=1}^{\infty} \bigcup_{\xi \in\left(A^{\prime} \cap D_{k}\right) \times\left(B^{\prime} \cap G_{k}\right)} \Omega_{\xi, k} .
$$

It is clear that $\Omega$ is a connected neighborhood of $X^{\prime}$. We will show that the functions $\widetilde{f}_{\xi, k}, \xi \in\left(A^{\prime} \cap D_{k}\right) \times\left(B^{\prime} \cap G_{k}\right), k \in \mathbb{N}$, can be glued together. We only need to check that $\widetilde{f}_{\xi, k}=\widetilde{f}_{\eta, k}$ on $\Omega_{\xi, k} \cap \Omega_{\eta, k} \backslash M, \xi=(a, b)$, $\eta=(c, d)$. Let $\varrho^{\prime}:=\varrho_{\xi, k}, \varrho^{\prime \prime}:=\varrho_{\eta, k}, f^{\prime}:=\widetilde{f}_{\xi, k}, f^{\prime \prime}:=\widetilde{f}_{\eta, k}$. Observe that

$$
\begin{aligned}
\Omega_{\xi, k} \cap \Omega_{\eta, k}= & \left(\Delta_{a}\left(\varrho^{\prime}\right) \times \Delta_{d}\left(\varrho^{\prime \prime}\right)\right) \cup\left(\Delta_{c}\left(\varrho^{\prime \prime}\right) \times \Delta_{b}\left(\varrho^{\prime}\right)\right) \\
& \cup\left(\left(\Delta_{a}\left(\varrho^{\prime}\right) \cap \Delta_{c}\left(\varrho^{\prime \prime}\right)\right) \times G_{k}\right) \cup\left(D_{k} \times\left(\Delta_{b}\left(\varrho^{\prime}\right) \cap \Delta_{d}\left(\varrho^{\prime \prime}\right)\right)\right) \\
= & : W_{1} \cup W_{2} \cup W_{3} \cup W_{4} .
\end{aligned}
$$

To prove that $f^{\prime}=f^{\prime \prime}$ on $W_{1} \backslash M$ it suffices to observe that $f^{\prime}=f^{\prime \prime}$ on $\left(A^{\prime} \cap \Delta_{a}\left(\varrho^{\prime}\right)\right) \times\left(B^{\prime} \cap \Delta_{d}\left(\varrho^{\prime \prime}\right)\right) \backslash M$ (and use Lemma 6). The same argument solves the problem on $W_{2} \backslash M$.

If $W_{3} \neq \emptyset$, then the equality holds on a non-empty set $W_{3} \cap W_{1} \backslash M$ and we only need to use the identity principle. The same argument works on $W_{4} \backslash M$.

STEP 6. Recall that the sets $A^{\prime}, B^{\prime}$ are locally regular and $A^{\prime}$ is a Borel set. Moreover, $h_{A^{\prime}, D}^{*}=h_{A, D}^{*}$ and $h_{B^{\prime}, G}^{*}=h_{B, G}^{*}$. Hence $\widehat{X}^{\prime}=\widehat{X}$.

First we prove that $\widehat{X}$ is the envelope of holomorphy of $\Omega$. We only need to show that any function $g \in \mathcal{O}(\Omega)$ extends holomorphically to $\widehat{X}$. Fix a $g \in \mathcal{O}(\Omega)$. By Theorem 4 (applied to the cross $X^{\prime}$ ), there exists a $\widehat{g} \in \mathcal{O}(\widehat{X})$ (recall that $\widehat{X}=\widehat{X}^{\prime}$ ) such that $\widehat{g}=g$ on $X^{\prime}$. By Lemma $6, \widehat{g}=g$ on $\Omega$. 
By Theorem 5 there exists a pure 1-dimensional analytic subset $\widehat{M}$ of $\widehat{X}$ such that for any $g \in \mathcal{O}(\Omega \backslash M)$ there exists a $\widehat{g} \in \mathcal{O}(\widehat{X} \backslash \widehat{M})$ with $\widehat{g}=g$ on $\Omega \backslash(M \cup \widehat{M})$. We also know that if $U=\widehat{X}$ and $M$ is pure 1-dimensional, then we can take $\widehat{M}=M$.

Now take an $f \in \mathcal{O}_{\mathrm{s}}(X \backslash M)$ and let $\tilde{f} \in \mathcal{O}(\Omega \backslash M)$ be such that $\tilde{f}=f$ on $X^{\prime} \backslash M$ (Step 5). Let $\widehat{f} \in \mathcal{O}(\widehat{X} \backslash \widehat{M})$ be such that $\widehat{f}=\tilde{f}$ in $\Omega \backslash(M \cup \widehat{M})$. In particular, $\widehat{f}=f$ on $X^{\prime} \backslash(M \cup \widehat{M})$. By Lemma $6, \widehat{f}=f$ on $X \backslash(M \cup \widehat{M})$.

Using Lemma 6 once again, we conclude that the function $\widehat{f}$ is uniquely determined.

Acknowledgements. We wish to thank the Niedersächsisches Ministerium für Wissenschaft und Kunst (Az.15.3-50 113(55) PL) and the Committee of Scientific Research (KBN), Warsaw (PB 2 P03A 017 14) for their financial support.

We also wish to thank Professor Siciak for calling our attention to the problem.

\section{References}

[Chi 1989] E. M. Chirka, Complex Analytic Sets, Kluwer, 1989.

[Jar-Pfl 2000] M. Jarnicki and P. Pflug, Extension of Holomorphic Functions, de Gruyter Expositions Math. 34, de Gruyter, 2000.

[Kli 1991] M. Klimek, Pluripotential Theory, Oxford Univ. Press, 1991.

[Ngu-Zer 1991] Nguyen Thanh Van and A. Zeriahi, Une extension du théorème de Hartogs sur les fonctions séparément analytiques, in: Analyse Complexe Multivariable, Récents Développements, A. Meril (ed.), EditEl, Rende, 1991, 183-194.

[Ökt 1998] O. Öktem, Extension of separately analytic functions and applications to range characterization of the exponential Radon transform, Ann. Polon. Math. 70 (1998), 195-213.

[Ökt 1999] - Extending separately analytic functions in $\mathbb{C}^{n+m}$ with singularities, in: Extension of separately analytic functions and applications to mathematical tomography (Thesis), Dept. Math., Stockholm Univ., 1999.

[Sic 2000] J. Siciak, Holomorphic functions with singularities on algebraic sets, Univ. Iagell. Acta Math. (2001), to appear.

Institute of Mathematics

Jagiellonian University

Reymonta 4

30-059 Kraków, Poland

E-mail: jarnicki@im.uj.edu.pl
Fachbereich Mathematik Carl von Ossietzky Universität Oldenburg Postfach 2503

D-26111 Oldenburg, Germany E-mail: pflug@mathematik.uni-oldenburg.de 